\title{
Natural Plasmodium infection in wild macaques of three states in peninsular Malaysia
}

\author{
Amirah Amir ${ }^{\mathrm{a}}$, Shahhaziq Shahari ${ }^{\mathrm{a}}$, Jonathan Wee Kent Liew ${ }^{\mathrm{a}}$, Jeremy Ryan de Silva ${ }^{\mathrm{a}}$, \\ Mohammad Behram Khan ${ }^{\mathrm{a}}$, Meng Yee Lai ${ }^{\mathrm{a}}$, Georges Snounou ${ }^{\mathrm{b}}$, Mohd Lutfi Abdullah ${ }^{\mathrm{c}}$, \\ Millawati Gani ${ }^{c}$, Jeffrine J. Rovie-Ryan ${ }^{c}$, Yee-Ling Lau ${ }^{\mathrm{a}, *}$ \\ ${ }^{a}$ Department of Parasitology Faculty of Medicine, University Malaya, 50603, Kuala Lumpur, Malaysia \\ ${ }^{\mathrm{b}}$ CEA-Université Paris Sud 11-INSERM U1184, Immunology of Viral Infections and Autoimmune Diseases (IMVA) IDMIT Department IBFJ DRF, Fontenay-aux-Roses, \\ France \\ ${ }^{\mathrm{c}}$ National Wildlife Forensic Laboratory, Ex-situ Conservation Division Department of Wildlife and National Parks Peninsular Malaysia, 56100, Kuala Lumpur, Malaysia
}

\section{A R T I C L E IN F O}

\section{Keywords:}

Malaria

Plasmodium

Macaque

Zoonosis

Malaysia

\begin{abstract}
A B S T R A C T
Zoonotic cases of Plasmodium knowlesi account for most malaria cases in Malaysia, and humans infected with $P$. cynomolgi, another parasite of macaques have recently been reported in Sarawak. To date the epidemiology of malaria in its natural Macaca reservoir hosts remains little investigated. In this study we surveyed the prevalence of simian malaria in wild macaques of three states in Peninsular Malaysia, namely Pahang, Perak and Johor using blood samples from 103 wild macaques (collected by the Department of Wildlife and National Parks Peninsular Malaysia) subjected to microscopic examination and nested PCR targeting the Plasmodium small subunit ribosomal RNA gene. As expected, PCR analysis yielded significantly higher prevalence (64/103) as compared to microscopic examination (27/103). No relationship between the age and/or sex of the macaques with the parasitaemia and the Plasmodium species infecting the macaques could be identified. Wild macaques in Pahang had the highest prevalence of Plasmodium parasites (89.7\%), followed by those of Perak (69.2\%) and Johor (28.9\%). Plasmodium inui and $P$. cynomolgi were the two most prevalent species infecting the macaques from all three states. Half of the macaques (33/64) harboured two or more Plasmodium species. These data provide a baseline survey, which should be extended by further longitudinal investigations that should be associated with studies on the bionomics of the anopheline vectors. This information will allow an accurate evaluation of the risk of zoonotic transmission to humans, and to elaborate effective strategies to control simian malaria.
\end{abstract}

\section{Introduction}

The global mortality of malaria estimated at 405,000 deaths in 2018 (WHO, 2019) is primarily due to infection by two of the four Plasmodium species that infect humans, primarily $P$. falciparum but also $P$. vivax. However, over the recent years the burden of clinical malaria in Malaysia is predominantly due to infection by $P$. knowlesi, a parasite of macaques. Although it had long been known that humans are susceptible to this species through experimental infections (Knowles and Das Gupta, 1932), only one single case of naturally acquired knowlesi malaria was recorded in the 1960's (Chin et al., 1965). The true zoonotic potential was only recognized with the discovery in 2004 of focus of zoonotic P. knowlesi in Sarawak (Singh et al., 2004).

Eight Macaca species in Southeast Asia are known to be the natural host of one or more of six species of Plasmodium: P. coatneyi, P. cynomolgi, $P$. fieldi, $P$. fragile, $P$. inui, $P$. knowlesi, and $P$. simiovale (Coatney et al., 1971). It is thought that recent environmental and social changes have contributed to increase the proximity of humans to wild macaques. Thus, deforestation and the associated exploitation of these new areas for agriculture has brought macaques and their anopheline vectors closer to the human habitats (Fornace et al., 2016). Furthermore, the rising popularity of eco-tourism has also brought humans closer to the natural habitat of macaques. Consequently, over the last decade a rising number of zoonotic knowlesi malaria cases are recorded in Malaysia, particularly in the states of Sarawak and Sabah in Borneo, (Barber et al., 2017). Furthermore since 2004, knowlesi malaria cases were reported in many of the Southeast Asian countries where macaques are endemic (Cox-Singh, 2012) and there are reports

\footnotetext{
* Corresponding author.

E-mail address: lauyeeling@um.edu.my (Y.-L. Lau).
} 